\title{
ON EXTENSION OF WRONSKIAN MATRICES
}

\section{DAVID M. KRABILL}

1. Introduction. By an interval $J$ we shall understand a finite interval of the type $a \leqq x \leqq b$. If $u_{1}(x), u_{2}(x), \cdots, u_{n}(x)$ are real functions possessing finite derivatives of the first $t$ orders in an interval $J$ and $0 \leqq s \leqq t$, we call the functional matrix

$$
M_{s}\left(u_{1}, \cdots, u_{n}\right) \equiv\left\|\begin{array}{cccc}
u_{1} & u_{2} & \cdots & u_{n} \\
u_{1}^{\prime} & u_{2}^{\prime} & \cdots & u_{n}^{\prime} \\
\cdot & \cdot & \cdots & \cdot \\
u_{1}^{(s)} & u_{2}^{(s)} & \cdots & u_{n}^{(s)}
\end{array}\right\|
$$

their Wronskian matrix of order $s$. The Wronskian $W\left(u_{1}, \cdots, u_{n}\right)$ is the determinant of the matrix $M_{n-1}\left(u_{1}, \cdots, u_{n}\right)$.

The principal result we obtain is that if $n \leqq s \leqq t$ and the Wronskian matrix of order $s$ for $n$ arbitrary functions $u_{1}(x), u_{2}(x), \cdots, u_{n}(x)$ of class $^{1} C^{(t)}$ in an interval $J$ has constant rank $n$, there exists a function $u_{n+1}(x)$ of class $C^{(t)}$ such that the extended matrix $M_{s}\left(u_{1}, \cdots, u_{n+1}\right)$ has constant rank $n+1$ in $J$. We employ a theorem of Curtiss ${ }^{2}$ which may be stated in the form:

Theorem C. If $u_{1}(x), u_{2}(x), \cdots, u_{n}(x)$ are functions of class $C^{(t)}$ in an interval $J$ and their Wronskian matrix of order $t$ has rank $n$ throughout $J$, then the Wronskian $W\left(u_{1}, \cdots, u_{n}\right)$ has at most $\cdot$ isolated zeros.

From the extension property of Wronskian matrices we obtain a sufficient condition, in terms of the rank of a certain functional matrix, that an arbitrary set of functions having suitable class properties be solutions of an ordinary homogeneous linear differential equation.

2. Lemmas. We first prove two lemmas.

LEMMA 1. If $\delta, c_{1}, c_{2}, \cdots, c_{n}$ are given constants with $\delta>0$, there exists a function $f(x)$ of class $C^{(n)}$ in the interval $-1 \leqq x \leqq 1$ which satisfies the conditions: (1) $|f(x)| \leqq \delta,-1 \leqq x \leqq 1 ;(2) f^{(i)}(-1)=f^{(i)}(1)=0$, $i=0,1, \cdots, n$; (3) $f(0)=0, f^{(i)}(0)=c_{i}, i=1,2, \cdots, n$. 1942.

Presented to the Society, September 10, 1942; received by the editors Augúst 25,

${ }^{1}$ Each function has continuous derivatives of the first $t$ orders at every point of $J$.

2 D. R. Curtiss, The vanishing of the Wronskian and the problem of linear dependence, Math. Ann. vol. 65 (1908) Theorem 4. 
Proof. Let $p(x)$ be the polynomial

$$
c_{1} x+\left(c_{2} / 2 !\right) x^{2}+\cdots+\left(c_{n} / n !\right) x^{n} .
$$

Then $p(0)=0, p^{(i)}(0)=c_{i}$ and for some $\beta$ with $0<\beta<1,|p(x)| \leqq \delta$, $-\beta \leqq x \leqq \beta$. Take $\alpha$ such that $0<\alpha<\beta$. Let

$$
\theta=(-1)^{n}(x+\alpha)^{n+1} /(x+\beta)^{2}, \quad \phi=-(x-\alpha)^{n+1} /(x-\beta)^{2},
$$

and let

$$
g_{1}(x) \equiv\left\{\begin{array} { l l } 
{ 0 , } & { x \leqq - \beta , } \\
{ e ^ { \theta } , } & { - \beta < x < - \alpha , } \\
{ 1 , } & { - \alpha \leqq x \leqq 0 , }
\end{array} \quad g _ { 2 } ( x ) \equiv \left\{\begin{array}{ll}
1, & 0 \leqq x \leqq \alpha, \\
e^{\phi}, & \alpha<x<\beta, \\
0, & \beta \leqq x .
\end{array}\right.\right.
$$

The $g$ 's are of class $C^{(n)}$ in their respective intervals of definition and $g_{1}^{(i)}(0)=g_{2}^{(i)}(0)$ for $i=0,1, \cdots, n$. Then the function

$$
f(x) \equiv\left\{\begin{array}{lr}
p(x) g_{1}(x), & -1 \leqq x \leqq 0, \\
p(x) g_{2}(x), & 0 \leqq x \leqq 1,
\end{array}\right.
$$

is of class $C^{(n)}$ in the interval $-1 \leqq x \leqq 1$ and satisfies the prescribed conditions.

Lemma 2. Given an interval $J$ and constants $a_{i}$ and $b_{i}, i=0,1, \cdots, n$ with $a_{0} b_{0}>0$, there exists a function $f(x)$ which satisfies the conditions: (1) $f(x)$ is nonzero and of class $C^{(n)}$ in $J$; (2) $f^{(i)}(a)=a_{i}, f^{(i)}(b)=b_{i}$, $i=0,1, \cdots, n$.

Proof. Let $\delta=1 / 3 \min \left(\left|a_{0}\right|,\left|b_{0}\right|, b-a\right)$. Take $f_{k}(x), k=1$, 2, of class $C^{(n)}$ in the interval $-1 \leqq x \leqq 1$ such that: $\left|f_{k}(x)\right| \leqq \delta,-1 \leqq x \leqq 1$; $f_{k}^{(i)}(-1)=f_{k}^{(i)}(1)=f_{k}(0)=0, i=0,1, \cdots, n ; f_{1}^{(i)}(0)=a_{i}, f_{2}^{(i)}(0)=b_{i}$, $i=1,2, \cdots, n$. Let

$$
\begin{array}{ll}
u(x) \equiv f_{1}((x-a) / \delta)+a_{0}, & a \leqq x \leqq a+\delta, \\
v(x) \equiv f_{2}((x-b) / \delta)+b_{0}, & b-\delta \leqq x \leqq b .
\end{array}
$$

Then $u(a)=u(a+\delta)=a_{0} ;|u(x)-u(a)| \leqq \delta, a \leqq x \leqq a+\delta ; u^{(i)}(a)=a_{i}$, $u^{(i)}(a+\delta)=0, i=1,2, \cdots, n$; and $v(b)=v(b-\delta)=b_{0} ;|v(x)-v(b)|$ $\leqq \delta, b-\delta \leqq x \leqq b ; v^{(i)}(b)=b_{i}, v^{(i)}(b-\delta)=0, i=1,2, \cdots, n$. Moreover, both $u(x)$ and $v(x)$ are nonzero in their respective mutually exclusive intervals of definition by our choice of $\delta$. Let $w(x) \equiv\left(a_{0}-b_{0}\right) e^{\theta}+b_{0}$, $a+\delta \leqq x \leqq b-\delta$, where $\theta=-(x-a-\delta)^{n+1} /(x-b+\delta)^{2}$. Then $w(x)$ is nonzero throughout its interval of definition and

$$
\begin{array}{r}
w(a+\delta)=a_{0}, w(b-\delta)=b_{0}, w^{(i)}(a+\delta)=w^{(i)}(b-\delta)=0, \\
i=1,2, \cdots, n .
\end{array}
$$


Therefore the function

$$
f(x) \equiv \begin{cases}u(x), & a \leqq x \leqq a+\delta \\ w(x), & a+\delta \leqq x \leqq b-\delta, \\ v(x), & b-\delta \leqq x \leqq b\end{cases}
$$

satisfies the conditions of the lemma.

3. Extension of Wronskian matrices. Let $u(x)$ be a function of class $C^{(t)}, t \geqq 1$, in an interval $J$ with $M_{1}(u)$ of rank 1 at every point of $J$. If $v(x)$ of class $C^{(t)}$ exists such that $M_{1}(u, v)$ has rank 2 throughout $J$, the Wronskian $W(u, v)$ is nonzero in $J$. Thus in this case there exists a function $K(x)$ of class $C^{(t-1)}$ and nonzero in $J$ such that $v(x)$ is a solution of the differential equation

$$
u(x) y^{\prime}-u^{\prime}(x) y=K(x) .
$$

This leads us to ask: does (1) have a solution of class $C^{(t)}$ in $J$ for arbitrary $K(x)$ of class $C^{(t-1)}$ which is nonzero in $J$ ? We give a counter-example.

Example. Let $J$ be the interval $0 \leqq x \leqq 2$ and take $u(x)=1-x$, $K(x)=1+|1-x|^{1 / 2}$. Then the differential equation (1) becomes

$$
(1-x) y^{\prime}+y=1+|1-x|^{1 / 2},
$$

for which the general solution in the interval $0 \leqq x<1$ is

$$
y(x)=u(x) \int_{0}^{x}\left(K(z) / u^{2}(z)\right) d z+c u(x)
$$

where $c$ is constant. Thus

$$
y^{\prime}(x)=(1 / u(x))\left[K(x)+u^{\prime}(x) y(x)\right] \equiv 3-c-(1-x)^{-1 / 2}
$$

and $\lim _{x \rightarrow 1-0} y^{\prime}(x)$ does not exist, so that the differential equation (2) has no solution of class $C^{\prime}$ defined in the interval $0 \leqq x \leqq 2$.

We now consider the special case of extending a Wronskian matrix of $n$ columns and $n+1$ rows which has rank $n$ throughout an interval $J$.

TheOREM 1. If $u_{1}(x), u_{2}(x), \cdots, u_{n}(x)$ are functions of class $C^{(t)}$ $(t \geqq n)$ in an interval $J$ and their Wronskian matrix of order $n$ has rank $n$ at every point of $J$, there exists a function $u_{n+1}(x)$ of class $C^{(t)}$ such that $M_{n}\left(u_{1}, \cdots, u_{n+1}\right)$ has rank $n+1$ throughout $J$.

Proof. Since $M_{n}\left(u_{1}, \cdots, u_{n}\right)$ has rank $n$ throughout $J$, it follows 
from Theorem $\mathrm{C}$ that $W\left(u_{1}, \cdots, u_{n}\right)$ has at most a finite number of zeros in $J$. Denote by

$$
f_{r}(x), \quad r=0,1, \cdots, n,
$$

the cofactor of $y^{(r)}$ in the determinant $W\left(u_{1}, \cdots, u_{n}, y\right)$ in which $y$ is unknown. If we prove the existence of a function $K(x)$ which is nonzero and of class $C^{(t-n)}$ in $J$ for which the differential equation

$$
f_{n}(x) y^{(n)}+f_{n-1}(x) y^{(n-1)}+\cdots+f_{1}(x) y^{\prime}+f_{0}(x) y=K(x)
$$

has a solution defined in $J$, we shall have proved our theorem since the coefficients in (4) are of class $C^{(t-n)}$. If $f_{n}(x)$ does not vanish in $J$, standard existence theorems apply for arbitrary $K(x)$.

Let $f_{n}(x)$ vanish in $J$. Since $f_{n}(x) \equiv W\left(u_{1}, \cdots, u_{n}\right)$, its zeros are finite in number. Assume that the zeros are at the points $c_{1}, c_{2}, \cdots, c_{m}$ with $c_{1}<c_{2}<\cdots<c_{m}$. If the $c$ 's are not all interior points of $J$, let

$$
U_{\imath}(x) \equiv \begin{cases}\sum_{j=0}^{t} \frac{1}{j !} u_{i}^{(j)}(a)(x-a)^{j}, & x<a, \\ u_{i}(x), & a \leqq x \leqq b, \quad i=1,2, \cdots, n . \\ \sum_{j=0}^{t} \frac{1}{j !} u_{i}^{(j)}(b)(x-b)^{j}, & x>b,\end{cases}
$$

Then $W\left(U_{1}, \cdots, U_{n}\right)$ is identical with $W\left(u_{1}, \cdots, u_{n}\right)$ in $J$, and is a polynomial for $x$ not in $J$. Therefore $W\left(U_{1}, \cdots, U_{n}\right)$ has isolated zeros and for some $a^{*}<a$ and $b^{*}>b$, the $c^{\prime}$ s are its only zeros in the interval $a^{*} \leqq x \leqq b^{*}$. Consequently we assume without loss of generality that $c_{1}>a$ and $c_{m}<b$.

Since $M_{n}\left(u_{1}, \cdots, u_{n}\right)$ has rank $n$ throughout $J$, for each $i$, $i=1,2, \cdots, m$, at least one function in (3) does not vanish at $c_{i}$. Denote by $f_{k_{i}}(x)$ a function from (3) such that $f_{k_{i}}\left(c_{i}\right) \neq 0, i=1,2, \cdots, m$. Define

$$
q_{i}(x) \equiv \pm\left(1 / k_{i} !\right)\left(x-c_{i}\right)^{k_{i}}, \quad i=1,2, \cdots, m,
$$

where the ambiguous sign is so chosen that each Wronskian $W\left(u_{1}, \cdots, u_{n}, q_{i}\right)$, which has the value $\pm f_{k_{i}}\left(c_{i}\right)$ at $c_{i}$, has the same sign at $c_{i}$. Then, for some $\epsilon>0$ with $a<c_{1}-\epsilon<c_{1}+\epsilon<c_{2}-\epsilon<\cdots$ $<c_{m}+\epsilon<b$, $Q_{i}(x) \equiv W\left(u_{1}, \cdots, u_{n}, q_{i}\right) \neq 0, c_{i}-\epsilon \leqq x \leqq c_{i}+\epsilon, i=1,2, \cdots, m$, and $q_{i}(x)$ is a solution of the differential equation

$$
W\left(u_{1}, \cdots, u_{n}, y\right)=Q_{i}(x), \quad i=1,2, \cdots, m .
$$


Denote the nterval

$$
c_{k-1}+\epsilon \leqq x \leqq c_{k}-\epsilon, k=1,2, \cdots, m+1,
$$

where $c_{0}+\epsilon=a$ and $c_{m+1}-\epsilon=b$, by $J_{k}$. Let $P_{k}(x)$ be any function (Lemma 2) which is nonzero and of class $C^{(t-n)}$ in $J_{k}$ and satisfies the conditions

$$
\begin{aligned}
P_{k}^{(j)}\left(c_{k-1}+\epsilon+0\right) & =Q_{k-1}^{(j)}\left(c_{k-1}+\epsilon-0\right), \\
P_{k}^{(j)}\left(c_{k}-\epsilon-0\right) & =Q_{k}^{(j)}\left(c_{k}-\epsilon+0\right),
\end{aligned}
$$

for $j=0,1, \cdots, t-n$, where

$$
\begin{aligned}
Q_{0}^{(j)}\left(c_{0}+\epsilon-0\right) & =Q_{1}^{(j)}\left(c_{1}-\epsilon+0\right), \\
Q_{m+1}^{(j)}\left(c_{m+1}-\epsilon+0\right) & =Q_{m}^{(j)}\left(c_{m}+\epsilon-0\right) .
\end{aligned}
$$

Then each function

$$
K_{k}(x) \equiv\left\{\begin{array}{ll}
P_{k}(x), & c_{k-1}+\epsilon \leqq x \leqq c_{k}-\epsilon, \\
Q_{k}(x), & c_{k}-\epsilon \leqq x \leqq c_{k}+\epsilon,
\end{array} \quad k=1,2, \cdots, m+1,\right.
$$

where $c_{m+1}+\epsilon=b$, is nonzero and of class $C^{(t-n)}$ in its interval of definition by the second set of conditions in (6). Thus the function $K(x)$ defined in $J$ by

(7) $K(x) \equiv K_{k}(x), \quad c_{k-1}+\epsilon \leqq x \leqq c_{k}+\epsilon, \quad k=1,2, \cdots, m+1$,

is nonzero and of class $C^{(t-n)}$ in $J$ by the first set of conditions in (6).

Since $f_{n}(x) \neq 0$ in $J_{1}$, the differential equation $W\left(u_{1}, \cdots, u_{n}, y\right)$ $=P_{1}(x)$ has a solution $p_{1}(x)$ which satisfies the initial conditions

$$
p_{1}^{(j)}\left(c_{1}-\epsilon-0\right)=q_{1}^{(j)}\left(c_{1}-\epsilon+0\right), \quad j=0,1, \cdots, n-1 .
$$

It follows from the second set of conditions in (6) that (8) also holds for $j=n, n+1, \cdots, t$. The function

$$
y_{1}(x) \equiv \begin{cases}p_{1}(x), & a \leqq x \leqq c_{1}-\epsilon, \\ q_{1}(x), & c_{1}-\epsilon \leqq x \leqq c_{1}+\epsilon,\end{cases}
$$

is therefore a solution of the differential equation $W\left(u_{1}, \cdots, u_{n}, y\right)$ $=K_{1}(x)$.

In $J_{2}$ the differential equation

$$
W\left(u_{1}, \cdots, u_{n}, y\right)=P_{2}(x)
$$

has a solution $p_{2}(x)$ with $p_{2}^{(j)}\left(c_{1}+\epsilon+0\right)=y_{1}^{(j)}\left(c_{1}+\epsilon-0\right), j=0,1, \cdots, t$, 
and also a solution $r(x)$ with $r^{(j)}\left(c_{2}-\epsilon-0\right)=q_{2}^{(j)}\left(c_{2}-\epsilon+0\right), j=0$, $1, \cdots, t$. Now the difference of any two solutions of (9) is a solution of the corresponding homogeneous equation for which the $u$ 's are linearly independent solutions. Thus, for some set of constants $a_{1}, a_{2}, \cdots, a_{n}$,

$$
p_{2}(x) \equiv r(x)+a_{1} u_{1}(x)+\cdots+a_{n} u_{n}(x), \quad c_{1}+\epsilon \leqq x \leqq c_{2}-\epsilon .
$$

Then the function

$$
y_{2}(x) \equiv \begin{cases}p_{2}(x), & c_{1}+\epsilon \leqq x \leqq c_{2}-\epsilon, \\ q_{2}(x)+a_{1} u_{1}(x)+\cdots+a_{n} u_{n}(x), & c_{2}-\epsilon \leqq x \leqq c_{2}+\epsilon,\end{cases}
$$

is of class $C^{(t)}$ in its interval of definition with

$$
y_{2}^{(j)}\left(c_{1}+\epsilon+0\right)=y_{1}^{(j)}\left(c_{1}+\epsilon-0\right), \quad j=0,1, \cdots, t,
$$

and is a solution of the differential equation $W\left(u_{1}, \cdots, u_{n}, y\right)=K_{2}(x)$.

We continue in the above manner, obtaining $y_{k}(x), c_{k-1}+\epsilon \leqq x \leqq c_{k}$ $+\epsilon(k=2,3, \cdots, m+1)$ of class $C^{(t)}$ in its interval of definition with

$$
y_{k}^{(j)}\left(c_{k-1}+\epsilon+0\right)=y_{k-1}^{(j)}\left(c_{k-1}+\epsilon-0\right), \quad j=0,1, \cdots, t,
$$

which is a solution of the differential equation $W\left(u_{1}, \cdots, u_{n}, y\right)$ $=K_{k}(x)$. Then the function $u_{n+1}(x)$ defined in $J$ by

$$
u_{n+1}(x) \equiv y_{k}(x), \quad c_{k-1}+\epsilon \leqq x \leqq c_{k}+\epsilon, \quad k=1,2, \cdots, m+1,
$$

is of class $C^{(t)}$ in $J$ and is a solution of (4) with $K(x)$ defined by (7).

In the following theorem we consider the general case of extending a Wronskian matrix.

THEOREM 2. If $u_{1}(x), u_{2}(x), \cdots, u_{n}(x)$ are functions of class $C^{(t)}$ $(t \geqq n)$ in an interval $J$ and their Wronskian matrix of order $s(n \leqq s \leqq t)$ has rank $n$ at every point of $J$, there exists a function $u_{n+1}(x)$ of class $C^{(t)}$ such that $M_{s}\left(u_{1}, \cdots, u_{n+1}\right)$ has rank $n+1$ throughout $J$.

Proof. The case $s=n$ is treated in Theorem 1. Assume now that $M_{n}\left(u_{1}, \cdots, u_{n}\right)$ does not have rank $n$ throughout $J$. Since $M_{s}\left(u_{1}, \cdots, u_{n}\right)$ has rank $n$ at every point, it follows from Theorem C that $W\left(u_{1}, \cdots, u_{n}\right)$ has isolated zeros. Define $f_{r}(x), r=0,1, \cdots, n$, as in (3). Since the $f^{\prime}$ s are the $n$-rowed minor determinants of $M_{n}\left(u_{1}, \cdots, u_{n}\right)$ they have at least one zero in common, but only a finite number for $f_{n}(x) \equiv W\left(u_{1}, \cdots, u_{n}\right)$. Assume that the common zeros of the $f$ 's are at the points $c_{1}, c_{2}, \cdots, c_{m}$ with $c_{1}<c_{2}<\cdots<c_{m}$. There is no loss of generality to assume that $c_{1}>a$ and $c_{m}<b$. 
For each $i, i=1,2, \cdots, m$, some $n$-rowed determinant of $M_{s}\left(u_{1}, \cdots, u_{n}\right)$, which we shall denote by $\Delta_{i}$, does not vanish at $c_{i}$. If $u(x)$ is a function of class $C^{(t)}$ in $J$ and row $r$ of $M_{s}\left(u_{1}, \cdots, u_{n}\right)$ is not a row of $\Delta_{i}$, denote by $\Delta_{i, r}$ the determinant of $M_{s}\left(u_{1}, \cdots, u_{n}, u\right)$ which consists of row $r$ and the rows represented in $\Delta_{i}$.

Let row $r_{i}$ be the first row of $M_{s}\left(u_{1}, \cdots, u_{n}\right)$ which is not a row of $\Delta_{i}$. Define

$$
q_{i}(x) \equiv\left(1 /\left(r_{i}-1\right) !\right)\left(x-c_{i}\right)^{r_{i}-1}, \quad i=1,2, \cdots, m .
$$

Then at $c_{i}$ the determinant $\Delta_{i, r_{i}}$ of $M_{s}\left(u_{1}, \cdots, u_{n}, q_{i}\right)$ has the value $(-1)^{n+1+r_{i} \Delta_{i}}\left(c_{i}\right)$, hence for some $\delta>0$ with $a<c_{1}-\delta<c_{1}+\delta<c_{2}-\delta$ $<\cdots<c_{m}+\delta<b$,

$$
\Delta_{i, r_{i}} \neq 0, c_{i}-\delta \leqq x \leqq c_{i}+\delta, \quad i=1,2, \cdots, m .
$$

Since $M_{s}\left(u_{1}, \cdots, u_{n}, q_{i}\right)$ has rank $n+1$ throughout the interval $c_{i}-\delta \leqq x \leqq c_{i}+\delta$ by $(10), W\left(u_{1}, \cdots, u_{n}, q_{i}\right)$ has isolated zeros in this interval. Thus, for some $\epsilon>0$ with $\epsilon \leqq \delta$,

$$
Q_{i}(x) \equiv W\left(u_{1}, \cdots, u_{n}, q_{i}\right) \neq 0 \text { at } x=c_{i} \pm \epsilon, i=1,2, \cdots, m .
$$

We shall assume that $Q_{i+1}\left(c_{i+1}-\epsilon\right), i=1,2, \cdots, m-1$, is positive or negative according as $Q_{i}\left(c_{i}+\epsilon\right)$ is positive or negative, since $q_{i+1}(x)$ could be replaced by $-q_{i+1}(x)$ and the expressions corresponding to (10) and (11) would hold.

Define the interval $J_{k}$ as in (5). Since $f_{0}(x), f_{1}(x), \cdots, f_{n}(x)$ have no common zero in $J_{k}, M_{n}\left(u_{1}, \cdots, u_{n}\right)$ has rank $n$ at every point of $J_{k}$. Thus, by the method employed in the proof of Theorem 1, we can define a function $P_{k}(x), k=1,2, \cdots, m+1$, which is nonzero and of class $C^{(t-n)}$ in $J_{k}$, which satisfies the conditions

$$
\begin{gathered}
P_{k}^{(j)}\left(c_{k-1}+\epsilon+0\right)=Q_{k-1}^{(j)}\left(c_{k-1}+\epsilon-0\right), \\
P_{k}^{(j)}\left(c_{k}-\epsilon-0\right)=Q_{k}^{(j)}\left(c_{k}-\epsilon+0\right)
\end{gathered}
$$

for $j=0,1, \cdots, t-n$, where

$$
\begin{aligned}
Q_{0}^{(j)}\left(c_{0}+\epsilon-0\right) & =Q_{1}^{(j)}\left(c_{1}-\epsilon+0\right), \\
Q_{m+1}^{(j)}\left(c_{m+1}-\epsilon+0\right) & =Q_{m}^{(j)}\left(c_{m}+\epsilon-0\right),
\end{aligned}
$$

and for which the differential equation $W\left(u_{1}, \cdots, u_{n}, y\right)=P_{k}(x)$, $k=1,2, \cdots, m+1$, has a solution of class $C^{(t)}$ defined in $J_{k}$.

In $J_{1}$ the differential equation

$$
W\left(u_{1}, \cdots, u_{n}, y\right)=P_{1}(x)
$$


has a solution $p_{1}(x)$ with $p_{1}^{(j)}\left(c_{1}-\epsilon-0\right)=q_{1}^{(j)}\left(c_{1}-\epsilon+0\right), j=0,1, \cdots, t$, in view of the second set of conditions in (12). Then the function

$$
y_{1}(x) \equiv \begin{cases}p_{1}(x), & a \leqq x \leqq c_{1}-\epsilon, \\ q_{1}(x), & c_{1}-\epsilon \leqq x \leqq c_{1}+\epsilon,\end{cases}
$$

is of class $C^{(t)}$, and $M_{s}\left(u_{1}, \cdots, u_{n}, y_{1}\right)$ has rank $n+1$ at every point of the interval $a \leqq x \leqq c_{1}+\epsilon$ since $p_{1}(x)$ is a solution of (13) and the determinant $\Delta_{1, r_{1}}$ does not vanish in the interval $c_{1}-\epsilon \leqq x \leqq c_{1}+\epsilon$ by (10).

In $J_{2}$ the differential equation

$$
W\left(u_{1}, \cdots, u_{n}, y\right)=P_{2}(x)
$$

has a solution $p_{2}(x)$ with $p_{2}^{(j)}\left(c_{1}+\epsilon+0\right)=y_{1}^{(j)}\left(c_{1}+\epsilon-0\right), j=0,1, \cdots, t$, and also a solution $r(x)$ with $r^{(j)}\left(c_{2}-\epsilon-0\right)=q_{2}^{(j)}\left(c_{2}-\epsilon+0\right), j=0$, $1, \cdots, t$. Since $u_{1}(x), u_{2}(x), \cdots, u_{n}(x)$ are linearly independent solutions of the homogeneous equation corresponding to (14), for some set of constants $a_{1}, a_{2}, \cdots, a_{n}$,

$$
p_{2}(x) \equiv r(x)+a_{1} u_{1}(x)+\cdots+a_{n} u_{n}(x), \quad c_{1}+\epsilon \leqq x \leqq c_{2}-\epsilon .
$$

Then the function

$$
y_{2}(x) \equiv \begin{cases}p_{2}(x), & c_{1}+\epsilon \leqq x \leqq c_{2}-\epsilon, \\ q_{2}(x)+a_{1} u_{1}(x)+\cdots+a_{n} u_{n}(x), & c_{2}-\epsilon \leqq x \leqq c_{2}+\epsilon,\end{cases}
$$

is of class $C^{(t)}$ in its interval of definition with

$$
y_{2}^{(j)}\left(c_{1}+\epsilon+0\right)=y_{1}^{(j)}\left(c_{1}+\epsilon-0\right), \quad j=0,1, \cdots, t,
$$

and $M_{s}\left(u_{1}, \cdots, u_{n}, y_{2}\right)$ has rank $n+1$ throughout the interval $c_{1}+\epsilon \leqq x \leqq c_{2}+\epsilon$, since $p_{2}(x)$ is a solution of (14) and the determinant $\Delta_{2, r_{2}}$ does not vanish in the interval $c_{2}-\epsilon \leqq x \leqq c_{2}+\epsilon$ by (10).

We continue in the above manner, obtaining $y_{k}(x), c_{k-1}+\epsilon \leqq x$ $\leqq c_{k}+\epsilon\left(k=2,3, \cdots, m+1\right.$, where $\left.c_{m+1}+\epsilon=b\right)$ of class $C^{(t)}$ in its interval of definition with

$$
y_{k}^{(1)}\left(c_{k-1}+\epsilon+0\right)=y_{k-1}^{(j)}\left(c_{k-1}+\epsilon-0\right), \quad j=0,1, \cdots, t,
$$

and with each matrix $M_{s}\left(u_{1}, \cdots, u_{n}, y_{k}\right)$ of rank $n+1$ throughout the interval $c_{k-1}+\epsilon \leqq x \leqq c_{k}+\epsilon$. Then the function $u_{n+1}(x)$ defined in $J$ by

$$
u_{n+1}(x) \equiv y_{k}(x), \quad c_{k-1}+\epsilon \leqq x \leqq c_{k}+\epsilon, \quad k=1,2, \cdots, m+1,
$$

is of class $C^{(t)}$ in $J$ and the Wronskian matrix $M_{s}\left(u_{1}, \cdots, u_{n+1}\right)$ has rank $n+1$ throughout $J$. 
4. Application to the theory of differential equations. The extension property of Wronskian matrices leads us to the following sufficient condition that a set of $n$ given functions be solutions of an ordinary homogeneous linear differential equation.

THEOREM 3. If $u_{1}(x), u_{2}(x), \cdots, u_{n}(x)$ are functions of class $C^{(t)}$ $(t \geqq n)$ in an interval $J$ and their Wronskian matrix of order $s(s<t)$ has rank $n$ at every point of $J$, then the $u$ 's are linearly independent solutions of a homogeneous linear differential equation of order $s+1$ of the type

$$
y^{(s+1)}+f_{s}(x) y^{(s)}+\cdots+f_{1}(x) y^{\prime}+f_{0}(x) y=0
$$

in which the $f_{i}(x)$ are functions of class $C^{(t-s-1)}$ in $J$.

Proof. Let $m=s-n+1$. The case $m=0$ is well known and $m<0$ is impossible. Assume now that $m>0$. Then $n \leqq s<t$ and, by $m$ successive applications of Theorem 2, there exist $m$ functions, $u_{n+1}(x), u_{n+2}(x), \cdots, u_{n+m}(x)$, of class $C^{(t)}$ in $J$ such that the Wronskian matrices

$$
M_{s}\left(u_{1}, \cdots, u_{n+1}\right), M_{s}\left(u_{1}, \cdots, u_{n+2}\right), \cdots, M_{s}\left(u_{1}, \cdots, u_{n+m}\right)
$$

have the respective ranks $n+1, n+2, \cdots, n+m$ at every point of $J$. Since $n+m=s+1$, the last matrix in (16) is the Wronskian matrix of order $s$ for $s+1$ functions of class $C^{(t)}(t>s)$ with $W\left(u_{1}, \cdots, u_{s+1}\right)$ $\neq 0$ throughout $J$. Therefore $u_{1}(x), u_{2}(x), \cdots, u_{s+1}(x)$ constitute a fundamental system of solutions of a homogeneous equation of type (15) with the $f_{i}(x)$ of class $C^{(t-s-1)}$ in $J$. Hence the $n$ given functions have the asserted property.

U.S.N.R. Midshipmen's School

New York City 